\title{
A genetic-Fuzzy Procedure for Solving Fuzzy Multiresponses Problem
}

\author{
Abbas Al-Refaie ${ }^{1 *}$, Rasha Abdullah ${ }^{2}$, Ghaith Bani Domi ${ }^{3}$
}

\begin{abstract}
Submitted: 29/03/2021x Accepted : 12/07/2021
Abstract: This research proposed a procedure that combines genetic algorithm (GA) technique and fuzzy goal programming to optimize process performance in experimental design for fuzzy multiple quality characteristics. Initially, regression models were formulated to relate each replicate of a quality characteristic with the process's controllable factors. The GA technique was then employed to determine the optimal factor settings for each response's replicate. The GA's optimal results were then deployed to develop a fuzzy regression model to relate fuzzy process settings with each quality characteristic. The fuzzy models were adopted to construct the fuzzy desirability and deviation matrices for all quality characteristics. Finally, three optimization models were developed to determine the lower, middle, and upper bounds of optimal factor settings. Three industrial applications, which were widely examined, were employed to illustrate the proposed procedure. Results revealed that the proposed GA-fuzzy procedure efficiently dealt with uncertainty in multiple quality characteristics and process settings by providing fuzzy optimal factor settings rather than crisp values. Such information can support process engineering in understanding the impact of variations/uncertainty on process and product performance and in deciding proper corrective and preventive actions. Compared to the Taguchi method, grey-Taguchi technique, and artificial neural networks approach, the proposed procedure is found efficient in optimizing process performance for multiple quality characteristics under uncertainty.
\end{abstract}

Keywords: Genetic algorithm, Desirability function, Fuzzy goal programming, Optimization

This is an open access article under the CC BY-SA 4.0 license. (https://creativecommons.org/licenses/by-sa/4.0/)

\section{Introduction}

To compete effectively in today's marketplace, firms must find ways to manufacture high-quality products at low-cost to meet or exceed customer expectations. Robust design proposed by Taguchi [1] has significantly enhanced product's quality and manufacturing processes' productivity at minimal experimentation costs and efforts. The designed experiments proposed by Taguchi [1] uses orthogonal arrays to study all the process factors with minimum number of experiments. Then, signal-to-noise ratio is used to determine the optimal factor settings of a manufacturing process and the most influential process factors that affect a single quality characteristic of a product or a process. This approach has been widely applied to optimize process performance in many business applications [2-3]. Nowadays, customer interests and product/process functionality require concurrent improvement of multiple quality characteristics of a product. The Taguchi method is a reliable method for optimizing a single quality response of main interest, while it primarily uses engineering judgment to identify the combination of optimal factor settings that enhance multiple quality characteristics [4-6]. This usually increases uncertainty in the decision-making process about the combination of optimal factor settings and does not guarantee concurrent improvement of multiple quality responses. Recently, process engineers should determine the optimal combination of process factor settings of a manufacturing process to enhance multiple quality characteristics of products simultaneously. Therefore,

\footnotetext{
${ }^{1}$ Department of Industrial Engineering, University of Jordan, Amman 11942, Jordan, ORCID ID: 0000-0002-3291-0805

${ }^{2}$ Received Master Degree in Industrial Engineering, University of Jordan, ORCID ID: 0000-0002-7500-4984

${ }^{3}$ Received Master Degree in Industrial Engineering, University of Jordan, ORCID ID: 0000-0001-8109-4587

* Corresponding Author Email: abbas.alrefai@ju.edu.jo
}

various optimization techniques were proposed in literature to deal with multiresponses problem in the Taguchi method; including the Taguchi methodology and neuro-fuzzy based model [7-9], genetic algorithm [10-12], grey-fuzzy logic Chiang [13], response surface methodology and Taguchi's technique [14], comparisons of efficiency between different systems technique in data envelopment analysis [15], fuzzy goal programming approach [16], Taguchi-based grey relational analysis [17-19], Taguchi methods, neural networks, desirability function, and genetic algorithms [20], particle swarm optimization [21], regression and neural network [22], neural networks and Taguchi method [23], Taguchi technique and upper bound technique [24], fuzzy neural network approach [25], Min-Max model in fuzzy goal programming [26], fuzzy goal programming-regression approach [27], multiple pentagon fuzzy responses [28], non-dominated sorting genetic algorithm II [29]. Nevertheless, most of these approached are deterministic optimization, which were carried out without considering the uncertainty due to measurement and process variations; therefore, the optimal solution will be sensitive to variations of input and process parameters. Hence, an appropriate procedure is required to deal with uncertainty in multiresponse problem. Further, customers require conforming product with all observed quality characteristics fall within specified specifications at minimum variability around the process mean and minimal shift of mean from the target. Such customer and process preferences are represented by ranges rather than crisp values. In summary, an effective optimization procedure must be developed to determine fuzzy multiresponses problem in experimental design. The Genetic Algorithm (GA) is an artificial intelligence search metaheuristic that that is particularly well suited to identify the optimal levels of input variables that results in the best/optimal conditions of output variables [30-35]. The algorithm starts by creating an initial population by randomly generating feasible solutions. Then, the sets of chromosomes pass through a self- 
development heuristic. The fitter individuals are then chosen to evolve through crossover and mutation. GA are a popular metaheuristic that is particularly well suited for this class of problems. The GA requires a fitness function, which represents the objective function and the fitness value that corresponds to the performance of an individual chromosome. To set the fitness function for GA problems, an objective function with a set of variables and constraints can be applied to maximize or minimize a given function. Population is a collection of individuals, which is normally randomly initialized. The two important aspects are the initial population generation and the population size. Furthermore, GA is uniquely distinguished by having a parallel populationbased search with stochastic selection of many individual solutions, stochastic crossover and mutation [35-36]. Selection is the process of choosing two parents from the population for crossing. Some of the various selection methods are stochastic uniform, remainder

roulette wheel selection, random selection, rank selection and tournament selection. In crossover, generally two chromosomes (parents) are combined together to generate new chromosomes (offspring). The parents are chosen with preference towards fitness so that offspring inherit good genes. By iteratively applying the crossover operator, genes of good chromosomes appear more frequently in the population, eventually leading to convergence to an overall optimal or near optimal solution. Two typical parameters must be determined, including crossover probability and crossover way. The crossover probabilities of 0.4 through 0.9 are generally proposed. The most common crossover ways, like single-point crossover, multipoint crossover, and uniform crossover, were adopted. In addition, three other crossover forms, including three-parent crossover, ordered crossover, and shuffle crossover. The mutation operator introduces random changes into characteristics of chromosomes, which provides genetic variety and enable the genetic algorithm to search a broader space. The different forms of mutation are constraint dependent, uniform and adaptive feasible. The purpose of mutation is to prevent GA from being trapped into local optimal solutions. A suitable mutation probability is specified according to the mutual of string length. That is, supposing the length of the genetic string consists of eight genes, the mutation probability is $1 / 8$. Flipping mutation is vastly applied to the enlarging solution spaces. Stopping criteria locates what causes the algorithm to terminate-generations, time limit, and fitness limit. The mutation operator consists of altering the genetic information of a member of the population. If the resulted individual has better fitness, it replaces the old individual. In this kind of algorithms, iterations are called "generations," which are processed until (1) a stop condition is meet or (2) the program reaches a predefined limit of generation. This algorithm is applicable to search for the solution of high degree of complexity that often involves attributes that are large, non-linear and discrete in nature. The objective of GA is to find the optimal settings of the input variables to the simulated system that makes the output variables at their best or optimal conditions. Traditional GA are customized to accommodate multi-objective problems by using specialized fitness functions and introducing methods to promote solution diversity [37-42]. However, the GA ignores the uncertainty in the observed measurements between the replicates of each quality characteristic, which may result in distinct optimal factor settings for each replicate of a quality characteristic. To solve this issue, the optimal factor settings for each replicate that are obtained by using GA can be further processed to determine a fuzzy combination of optimal process factor settings for each quality characteristic and/or multiple quality characteristics. An appropriate technique to achieve this objective is the fuzzy goal programming (FGP) technique, which was widely used in optimizing performance for several business applications [26-28]. The FGP utilizes the fuzzy regression models, pay-off matrices, and desirability function to transform multiple objectives into a single equivalent objective function with the consideration of overall desirability. The combined genetic-FGP procedure can identify the fuzzy optimal factor settings to enhance multiple quality responses of a product simultaneously. In this context, this research develops a genetic-fuzzy procedure for optimizing the process performance with multiple quality characteristics under uncertainty. The remaining of this research is outlined as follows. Section two presents the proposed procedure. Section three provides three case studies for procedure illustration. Section four discusses research results. Section five summarizes research conclusions.

\section{Proposed Genetic-Fuzzy (G-F) Procedure}

Assume there are $Q$ responses to enhances by optimizing a process of $J$ controllable factors. Suppose that the experimental work was repeated, which resulted in having $K$ replicate values of each response. The proposed G-F procedure is depicted in Fig. 1 and is described as follows:

Step 1: Let $y_{r k}(x)$ denotes regression model of replicate $k ; k=1, \ldots$, $K$, for quality characteristic $r ; r=1, \ldots, Q$, formulated as a function of $J$ process factors, $x_{f} ;=1, \ldots, J$. Formulate $y_{r k}(x)$ as follows:

$y_{r k}(x)=\beta_{0 k}+\sum_{f=1}^{v} \beta_{f k} x_{f}+\sum_{f=1}^{J} \beta_{f f k} x_{f}^{2}+\sum_{g<f} \sum \beta_{f g k} x_{f} x_{g}+\varepsilon ; \forall k, \forall r$

where $\beta_{0 k}$ is the intercept, and $\beta_{f k}, \beta_{f g k}$, and $\beta_{f f k}$ are the crisp coefficients of process factors in the regression model.

Step 2: Formulate an optimization model for each response's replicate utilizing Eq. (1) and a set of constraints on process settings. Use the GA technique to determine the optimal settings of controllable process factors. Repeat this step for all responses' replicates.

Step 3: Formulate the fuzzy multiple regression model, $\tilde{y}_{r}(\tilde{x})$, for the response $r$ using the regression coefficients of $y_{r k}(x)$ from all response replicates. Let $\tilde{\beta}_{0}, \tilde{\beta}_{f f}$, and $\tilde{\beta}_{f g}$ be by fuzzy number of regression coefficients. Then, the $\tilde{y}_{r}(\tilde{x})$ can be expressed as stated in Eq. (2).

$\tilde{y}_{r}(\tilde{x})=\tilde{\beta}_{0}+\sum_{f=1}^{J} \tilde{\beta}_{f} \tilde{x}_{f}+\sum_{f=1}^{J} \tilde{\beta}_{f f} \tilde{x}_{f}^{2}+\sum_{\mathrm{g}<f} \sum \tilde{\beta}_{f g} \tilde{x}_{f} \tilde{x}_{g}+\varepsilon \quad \forall r, \forall f$

where $\tilde{\beta}\left(\beta^{l}, \beta^{m}, \beta^{u}\right)$ is obtained using Eq. (3).

$\tilde{\beta}=\left(\beta^{\mathrm{m}}=\right.$ Average $\left.\beta, \beta^{1}=\left(\beta^{\mathrm{m}}-\delta \mathrm{s}\right), \beta^{\mathrm{u}}=\left(\beta^{\mathrm{m}}+\delta \mathrm{s}\right)\right)$

The $\delta$ is a constant value set by process engineers based on knowledge of allowable variability in process factor levels. The $s$ is the estimated standard of the values of the $\beta$ coefficient in regression models of all replicates of a quality characteristic. Formulate the $\tilde{y}_{r}(\tilde{x})$ for all quality characteristics.

Step 4: Let $\tilde{x}_{q}$ denotes the fuzzy optimal factor settings for the $q$ th response; $q \in Q$, and $y_{r}\left(\tilde{x}^{q}\right)$ be the value of response $r$ resulted from substituting the values of $\tilde{x}_{q}$. Construct the $y_{r}\left(\tilde{x}^{q}\right)$ matrix as displayed in Table 1. 
Table 1. The $y_{r}\left(\tilde{x}^{q}\right)$ matrix.

\begin{tabular}{|c|ccccc|}
\hline$\tilde{x}_{q}$ & $\tilde{y}_{1}\left(\tilde{x}^{q}\right)$ & $\ldots$ & $\tilde{y}_{r}\left(\tilde{x}^{q}\right)$ & $\ldots$ & $\tilde{y}_{Q}\left(\tilde{x}^{q}\right)$ \\
\hline$\tilde{x}^{1}$ & $\tilde{y}_{1}\left(\tilde{x}^{1}\right)$ & $\ldots$ & $\tilde{y}_{r}\left(\tilde{x}^{1}\right)$ & $\ldots$ & $\tilde{y}_{Q}\left(\tilde{x}^{1}\right)$ \\
$\cdot$ & $\cdot$ & $\cdot$ & $\cdot$ & $\cdot$ & $\cdot$ \\
$\cdot$ & $\cdot$ & $\cdot$ & $\cdot$ & $\cdot$ & $\cdot$ \\
$\cdot$ & $\cdot$ & $\cdot$ & $\cdot$ & $\cdot$ & $\cdot$ \\
$\tilde{x}^{Q}$ & $\tilde{y}_{1}\left(\tilde{x}^{Q}\right)$ & $\ldots$ & $\tilde{y}_{r}\left(\tilde{x}^{Q}\right)$ & $\ldots$ & $\tilde{y}_{Q}\left(\tilde{x}^{Q}\right)$ \\
\hline
\end{tabular}

Step 5: Formulate the fuzzy desirability function, $\tilde{s}_{r}\left(\tilde{y}_{r}(\tilde{x})\right)$, of $\tilde{y}_{r}(\tilde{x})$ depending on the type of the quality characteristic. Mathematically, the $\tilde{s}_{r}\left(\tilde{y}_{r}(\tilde{x})\right)$ functions for the nominal-the-best (NTB) response is expressed as stated in Eq. (4).

$$
\tilde{s}_{r}\left(\tilde{y}_{r}(\tilde{x})\right)= \begin{cases}\frac{\tilde{y}_{r}(\tilde{x})-\tilde{y}_{\min }}{\tilde{G}-\tilde{y}_{\min }}, & \tilde{y}_{\min } \leq \tilde{y}_{r}\left(\tilde{x}^{q}\right) \leq \tilde{G} \\ \frac{\tilde{y}_{r}(\tilde{x})-\tilde{y}_{\max }}{\tilde{G}-\tilde{y}_{\max }}, & \tilde{G} \leq \tilde{y}_{r}\left(\tilde{x}^{q}\right) \leq y_{\max } \\ 0, & \tilde{y}_{r}\left(\tilde{x}^{q}\right) \geq \tilde{y}_{\max } \text { or } \tilde{y}_{r}\left(\tilde{x}^{q}\right) \leq \tilde{y}_{\min }\end{cases}
$$

where $\tilde{G}$ is the nominal response value, and $\tilde{y}_{\min }$ and $\tilde{y}_{\max }$ are the minimal and maximal specified response values, respectively. Further, the $\tilde{s}_{r}\left(\tilde{y}_{r}(\tilde{x})\right)$ functions for larger-the-better (LTB) and smaller the better (STB) type responses are given in Eqs. (5) and (6), respectively.

$$
\begin{aligned}
& \tilde{s}_{r}\left(\tilde{y}_{r}(\tilde{x})\right)= \begin{cases}0, & \tilde{y}_{r}\left(\tilde{x}^{q}\right) \leq \tilde{y}_{\text {min }} \\
\tilde{y}_{r}(\tilde{x})-\tilde{y}_{\min }-\tilde{y}_{\min } & \tilde{y}_{\text {min }} \leq \tilde{y}_{r}\left(\tilde{x}^{q}\right) \leq \tilde{y}_{\max } \\
1, & \tilde{y}_{r}\left(\tilde{x}^{q}\right) \geq \tilde{y}_{\max }\end{cases} \\
& \tilde{s}_{r}\left(\tilde{y}_{r}(\tilde{x})\right)= \begin{cases}1, & \tilde{y}_{r}\left(\tilde{x}^{q}\right) \leq \tilde{y}_{\min } \\
\frac{\tilde{y}_{r}(\tilde{x})-\tilde{y}_{\max }}{\tilde{y}_{\text {min }}-\tilde{y}_{\max }}, & \tilde{y}_{\text {min }} \leq \tilde{y}_{r}\left(\tilde{x}^{q}\right) \leq \tilde{y}_{\max } \\
0, & \tilde{y}_{r}\left(\tilde{x}^{q}\right) \geq \tilde{y}_{\max }\end{cases}
\end{aligned}
$$

Construct the matrix of $\tilde{s}_{r}\left(\tilde{y}_{j}\left(\tilde{x}^{q}\right)\right)$ values as shown in Table 2 .

Table 2. The $\tilde{s}_{r}\left(\tilde{y}_{j}\left(\tilde{x}^{q}\right)\right)$ matrix.

\begin{tabular}{|c|c|c|c|c|c|}
\hline & $\tilde{s}_{1}\left(\tilde{y}_{1}\left(\tilde{x}^{q}\right)\right)$ & $\ldots$ & $\tilde{s}_{r}\left(\tilde{y}_{r}\left(\tilde{x}^{q}\right)\right)$ & $\ldots$ & $\tilde{s}_{Q}\left(\tilde{y}_{Q}\left(\tilde{x}^{q}\right)\right)$ \\
\hline$\tilde{x}^{1}$ & $\tilde{s}_{1}\left(\tilde{y}_{1}\left(\tilde{x}^{1}\right)\right)$ & $\ldots$ & $\tilde{s}_{r}\left(\tilde{y}_{r}\left(\tilde{x}^{1}\right)\right)$ & $\ldots$ & $\tilde{s}_{Q}\left(\tilde{y}_{Q}\left(\tilde{x}^{1}\right)\right)$ \\
\hline$\vdots$ & $\vdots$ & $\vdots$ & $\vdots$ & $\vdots$ & $\vdots$ \\
\hline$\tilde{x}^{Q}$ & $\tilde{s}_{1}\left(\tilde{y}_{1}\left(\tilde{x}^{Q}\right)\right)$ & $\ldots$ & $\tilde{s}_{r}\left(\tilde{y}_{r}\left(\tilde{x}^{Q}\right)\right)$ & $\cdots$ & $\tilde{s}_{Q}\left(\tilde{y}_{Q}\left(\tilde{x}^{o}\right)\right)$ \\
\hline
\end{tabular}

Let

$$
\tilde{p}_{r}=\tilde{s}_{r}\left(\tilde{y}_{r}\left(\tilde{x}^{r}\right)\right), r=1, \ldots, Q
$$

and

$$
\tilde{w}_{r}=\operatorname{Min}\left\{\tilde{s}_{r}\left(\tilde{y}_{r}\left(\tilde{x}^{1}\right)\right), \ldots, \tilde{s}_{r}\left(\tilde{y}_{r}\left(\tilde{x}^{Q}\right)\right)\right\}, \quad r=1, \ldots, Q
$$

Identify the values of $\tilde{p}_{r}$ and $\tilde{w}_{r}$.

Step 6: Develop the deviation function, $\tilde{S}_{r}\left(\tilde{y}_{r}\left(\tilde{x}^{q}\right)\right)$, using Eq. (9).

$\tilde{S}_{r}\left(\tilde{y}_{r}(\tilde{x})\right)=\frac{\tilde{y}_{r}^{u}(\tilde{x})-\tilde{y}_{r}^{m}(\tilde{x})}{1-\lambda}, \quad r=1, \ldots, Q$ where $\lambda$ has a value range between zero and one. Calculate and then list the $\tilde{S}_{r}\left(\tilde{y}_{r}\left(\tilde{x}^{q}\right)\right)$ values as shown in Table 3 .

Calculate the values of $\tilde{S}_{r}\left(\tilde{y}_{r}\left(\tilde{x}^{q}\right)\right)$ values then determine the values of $\tilde{P}_{r}$ and $\tilde{W}_{r}$ as states in Eqs. (10) and (11), respectively.

$\tilde{P}_{r}=\tilde{S}_{r}\left(\tilde{y}_{r}\left(\tilde{x}^{r}\right)\right), \quad r=1, \ldots, Q$

$\tilde{W}_{r}=\operatorname{Max}\left\{\tilde{S}_{r}\left(\tilde{y}_{r}\left(\tilde{x}^{1}\right), \ldots, \tilde{S}_{r}\left(\tilde{y}_{r}\left(\tilde{x}^{q}\right)\right)\right\}, r=1, \ldots, Q\right.$

Table 3. The $\tilde{S}_{r}\left(\tilde{y}_{r}\left(\tilde{x}^{q}\right)\right)$ matrix.

\begin{tabular}{|c|c|c|c|c|c|}
\hline$\tilde{x}^{q}$ & $\tilde{S}_{1}\left(\tilde{y}_{1}\left(\tilde{x}^{q}\right)\right)$ & $\ldots$ & $\tilde{S}_{r}\left(\tilde{y}_{r}\left(\tilde{x}^{q}\right)\right)$ & $\ldots$ & $\tilde{S}_{Q}\left(\tilde{y}_{Q}\left(\tilde{x}^{q}\right)\right)$ \\
\hline$\tilde{x}^{1}$ & $\tilde{S}_{1}\left(\tilde{y}_{1}\left(\tilde{x}^{1}\right)\right)$ & $\ldots$ & $\tilde{S}_{r}\left(\tilde{y}_{r}\left(\tilde{x}^{1}\right)\right)$ & $\ldots$ & $\tilde{S}_{Q}\left(\tilde{y}_{Q}\left(\tilde{x}^{1}\right)\right)$ \\
\hline$\vdots$ & $\vdots$ & $\vdots$ & $\vdots$ & $\vdots$ & $\vdots$ \\
\hline$\tilde{x}^{Q}$ & $\tilde{S}_{1}\left(\tilde{y}_{1}\left(\tilde{x}^{Q}\right)\right)$ & $\ldots$ & $\tilde{S}_{r}\left(\tilde{y}_{r}\left(\tilde{x}^{Q}\right)\right)$ & $\ldots$ & $\tilde{S}_{Q}\left(\tilde{y}_{Q}\left(\tilde{x}^{Q}\right)\right)$ \\
\hline
\end{tabular}

Step 7: Formulate a two-objective optimization model as follows:

$$
\begin{aligned}
& \operatorname{Max}\left\{\tilde{s}_{1}\left(\tilde{y}_{1}(\tilde{x})\right), \ldots, \tilde{s}_{r}\left(\tilde{y}_{r}(\tilde{x})\right)\right\} \\
& \operatorname{Min}\left\{\tilde{S}_{1}\left(\tilde{y}_{1}(\tilde{x})\right), \ldots, \tilde{S}_{Q}\left(\tilde{y}_{Q}(\tilde{x})\right)\right\} \\
& \text { s.t } \\
& x \in[\text { Factor settings }]
\end{aligned}
$$

The optimization models with two objective functions can transformed into a single-objective optimization model as follows. Let $\tilde{Z}_{r}\left(\tilde{y}_{r}(\tilde{x})\right)$ and $\tilde{T}_{r}\left(\tilde{y}_{r}(\tilde{x})\right)$ are two fuzzy functions indicating the degrees of satisfaction from desirability and robustness, respectively, and are defined as follows:

$$
\tilde{Z}_{r}\left(\tilde{y}_{r}(\tilde{x})\right)=\left(Z_{r}^{l}, Z_{r}^{m}, Z_{r}^{u}\right)
$$

and

$$
\tilde{T}\left(\tilde{y}_{r}(\tilde{x})\right)=\left(T_{r}^{l}, T_{r}^{m}, T_{r}^{u}\right)
$$

Then, $\tilde{Z}_{r}\left(\tilde{y}_{r}(\tilde{x})\right)$ and $\tilde{T}_{r}\left(\tilde{y}_{r}(\tilde{x})\right)$ functions are formulated as shown in Eqs. (14) and (15), respectively.

$\tilde{Z}_{r}\left(\tilde{y}_{r}(\tilde{x})\right)= \begin{cases}0, & \tilde{s}_{r}\left(\tilde{y}_{r}\left(\tilde{x}^{r}\right)\right) \leq \tilde{w}_{r} \\ \left.\frac{\tilde{s_{r}}(\tilde{y}}{\tilde{y}_{r}}(\tilde{x})\right)-\tilde{L}_{r} & , \quad \tilde{w}_{r} \leq \tilde{w_{r}}\left(\tilde{y}_{r}\left(\tilde{x}^{r}\right)\right) \leq \tilde{p}_{r} \\ 1, & \tilde{s}_{r}\left(\tilde{y}_{r}\left(\tilde{x}^{r}\right)\right) \geq \tilde{p}_{r}\end{cases}$

$\tilde{T_{r}}\left(\tilde{y_{r}}(\tilde{x})\right)= \begin{cases}1, & \tilde{S}_{r}\left(\tilde{y}_{r}\left(\tilde{x}^{r}\right)\right) \leq \tilde{P}_{r} \\ \frac{\tilde{W}_{r}-\tilde{S_{r}}\left(\tilde{y_{r}}(\tilde{x})\right)}{\tilde{W}_{r}-\tilde{P_{r}},}, \quad \tilde{P_{r}} \leq \tilde{S}_{r}\left(\tilde{y}_{r}\left(\tilde{x}^{r}\right)\right) \leq \tilde{W_{r}} \\ 0, & \tilde{S}_{r}\left(\tilde{y}_{r}\left(\tilde{x}^{r}\right)\right) \geq \tilde{W}_{r}\end{cases}$

To maximize the minimum degree of satisfaction from two objectives, let:

$\operatorname{Min} \tilde{Z}_{r}\left(\tilde{y}_{r}(\tilde{x})\right)=\tilde{Z}$

and

$\operatorname{Min} \tilde{T}_{r}\left(\tilde{y}_{r}(\tilde{x})\right)=\tilde{T}$

Finally, let $a_{1}$ and $a_{2}$ be the weights for desirability and robustness. The final optimization model will be expressed as: 
$\operatorname{Max} \quad a_{1} \tilde{Z}+a_{2} \tilde{T}$

s.t

$\tilde{s}_{r}(x)-\tilde{Z}\left(\tilde{p}_{r}-\tilde{w}_{r}\right) \geq \tilde{w}_{r} \quad, \quad r=1, \ldots, Q$

$\tilde{S}_{r}(x)+\tilde{T}\left(\tilde{W}_{r}-\tilde{P}_{r}\right) \leq \tilde{P}_{r} \quad, r=1, \ldots, Q$

$a_{1}+a_{2}=1$

$0 \leq \tilde{Z} \leq 1$

$0 \leq \tilde{T} \leq 1$

$x \in[$ Factor Levels].

The fuzzy optimization model shown in model (18) is expressed by three models: lower, middle, and upper denoted by $l, m$, and $u$, respectively. Obtain the fuzzy optimal levels, $\tilde{x}^{*}=\left(x_{1}^{l, m, u}, \ldots, x_{J}^{l, m, u}\right)$, of the controllable process factors. Then, estimate the fuzzy response values, $\tilde{y}^{*}=\left(y^{l}, y^{m}, y^{u}\right)$, at the fuzzy optimal process settings.

Step 8: Apply the proposed procedure on a manufacturing process and then compare the anticipated improvements in the multiple quality characteristics obtained using the proposed genetic-fuzzy procedure and the previously employed approaches.
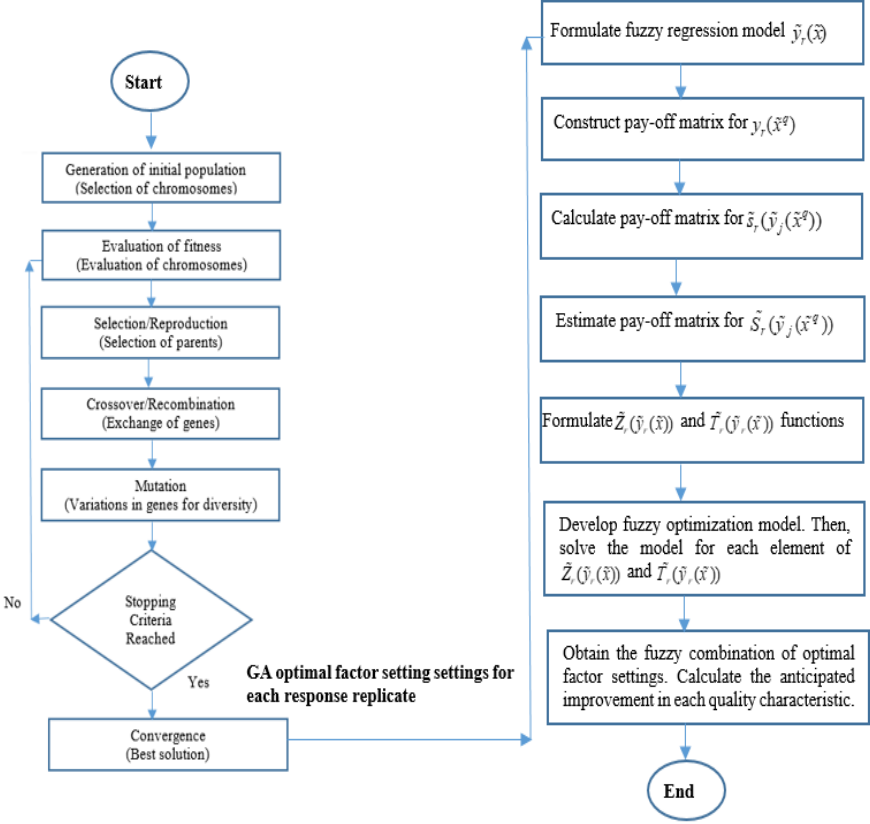

Fig.1. Depiction of the proposed genetic-fuzzy procedure.

\section{Illustrations}

Three widely studied case studies on the applications of the Taguchi method are provided for illustrating the proposed procedure and presented as follows.

\section{Case I: WEDM process}

This case study [5] aimed to optimize the performance of WEDM process for two important responses; $y_{1}$ : material removal rate ( $\mathrm{mm}^{2} / \mathrm{min}$, MRR, LTB) and $y_{2}$ : surface roughness $\left(\mathrm{mm}^{2} / \mathrm{min}, \mathrm{SR}\right.$, STB). Let the $y_{11}$ and $y_{12}$ represent the first and second replicate of MRR, $y_{1}$, respectively. Let the $\mathrm{y}_{21}$ and $\mathrm{y}_{22}$ represent the first and second replicate of SR, $y_{2}$, respectively. Four controllable process factors: the pulse on time $\left(x_{1}\right)$, delay time $\left(x_{2}\right)$, wire feed speed $\left(x_{3}\right)$, and ignition current $\left(x_{4}\right)$, were examined utilizing the L9 array shown in Table 4.
Table 4: Experimental data for WEDM process.

\begin{tabular}{|c|c|c|c|c|c|c|c|c|c|}
\hline $\begin{array}{c}\text { Ex. } \\
i\end{array}$ & \multicolumn{4}{|c|}{ Control factor } & \multicolumn{3}{c|}{$\begin{array}{c}\text { MRR } \\
\left(\mathrm{mm}^{2} / \mathrm{min}\right)\end{array}$} & \multicolumn{2}{c|}{$\begin{array}{c}\text { SR } \\
\left(\mathrm{mm}^{2} / \mathrm{min}\right)\end{array}$} \\
\hline & $x_{1}$ & $x_{2}$ & $x_{3}$ & $x_{4}$ & & $y_{i 11}$ & $y_{i 12}$ & $y_{i 21}$ & $y_{i 22}$ \\
\hline 1 & 0.6 & 4 & 8 & 8 & & 46 & 46 & 3.2 & 3.1 \\
\hline 2 & 0.6 & 6 & 12 & 12 & & 48 & 47 & 3.3 & 3.2 \\
\hline 3 & 0.6 & 8 & 15 & 16 & & 42 & 41 & 3.3 & 3.3 \\
\hline 4 & 0.8 & 4 & 12 & 16 & & 56 & 55 & 3.8 & 3.7 \\
\hline 5 & 0.8 & 6 & 15 & 8 & & 50 & 49 & 3.4 & 3.5 \\
\hline 6 & 0.8 & 8 & 8 & 12 & & 52 & 53 & 3.2 & 3.3 \\
\hline 7 & 1.2 & 4 & 15 & 12 & & 70 & 71 & 4.2 & 4 \\
\hline 8 & 1.2 & 6 & 8 & 16 & & 74 & 73 & 3.8 & 3.5 \\
\hline 9 & 1.2 & 8 & 12 & 8 & & 64 & 64 & 3.4 & 3.3 \\
\hline
\end{tabular}

Initially, the multiple regression models were formulated for each of the four response replicates. For illustration, the regression model for $y_{11}$ is expressed as:

$$
\begin{array}{r}
y_{11}=-2.80+41.50 x_{1}+4.30 x_{2}-1.25 x_{3}+3.90 x_{4} \\
+0.13 x_{2} x_{3}-0.58 x_{2}^{2}-0.14 x_{4}^{2}
\end{array}
$$

Further, the controllable factors; $x_{1}, \ldots, x_{4}$, are decided the following acceptable operating ranges of based on experimental knowledge:

$$
0.6 \leq x_{1} \leq 1.2 \quad 4 \leq x_{2} \leq 8 \quad 8 \leq x_{3} \leq 15 \quad 8 \leq x_{4} \leq 16
$$

The GA technique (selection-stochastic uniform; cross-over fraction of 0.6; Mutation- uniform and ratio of 0.5; cross-overheuristic and ratio of 1.4; Migration-forward) was then solved to determine optimal factor levels for each replicate as shown in Table 5.

Table 5. The GA optimal factor settings for WEDM process.

\begin{tabular}{|c|cc|cc|}
\hline \multirow{2}{*}{ Factor } & \multicolumn{2}{|c|}{$\boldsymbol{y}_{\mathbf{1}}$} & \multicolumn{2}{c|}{$\boldsymbol{y}_{\mathbf{2}}$} \\
& $y_{11}$ & $y_{12}$ & $y_{21}$ & $y_{22}$ \\
\hline$x_{1}{ }^{*}$ & 0.60 & 0.60 & 0.83 & 0.96 \\
$x_{2}{ }^{*}$ & 4.01 & 4.01 & 7.99 & 7.99 \\
$x{ }^{*}$ & 14.99 & 14.99 & 8.09 & 8.13 \\
$\mathbf{x}_{4}{ }^{*}$ & $\mathbf{8 . 0 1}$ & $\mathbf{8 . 0 1}$ & $\mathbf{8 . 0 0}$ & $\mathbf{8 . 0 0}$ \\
\hline
\end{tabular}

Utilizing the optimal factor settings in Table 5, the fuzzy optimal factor levels, $\tilde{x}^{1}$, for $y_{1}$ is expressed as

$$
\begin{aligned}
& \tilde{x}^{1}=\left\{\tilde{x}_{1}=(0.60,0.60,0.60) ; \tilde{x}_{2}=(4.01,4.01,4.01) ; \tilde{x}_{3}=\right. \\
& \left.(14.99,14.99,14.99) ; \tilde{x}_{4}=(8.01,8.01,8.01)\right\}
\end{aligned}
$$

Then, the fuzzy regression, $\tilde{y}_{1}(\tilde{x})$ for $y_{1}$ is then formulated respectively as follows:

$$
\begin{aligned}
& \tilde{y}_{1_{1}}(\tilde{x})=(-2.72,-2.69,-2.65)+(41.79,41.88,41.97) \tilde{x}_{1}+ \\
& (2.65,2.94,3.29) \tilde{x}_{2}+(-1.22,-1.21,-1.20) \tilde{x}_{3}+ \\
& (4.35,4.47,4.59) \tilde{x}_{4}+(-0.48,-0.46,-0.43) \tilde{x}_{2}^{2}+ \\
& (0.12,0.12,0.12) \tilde{x}_{2} \tilde{x}_{3}+(-0.17,-0.17,-0.16) \tilde{x}_{2} \tilde{x}_{4} .
\end{aligned}
$$

The fuzzy minimal, $\tilde{y}_{1 \min }$, and maximal, $\tilde{y}_{1 \max }$, acceptable ranges for $y_{1}$ (LTB) were set the fuzzy numbers $(37,37,37)$ and $(65,65$, $65)$, respectively. Then, the desirability function $\tilde{s}_{1}\left(\tilde{y}_{1}(\tilde{x})\right)$ is formulated as:

$$
\tilde{s}_{1}\left(\tilde{y}_{1}(\tilde{x})\right)= \begin{cases}0, & \tilde{y}_{1}\left(\tilde{x}^{q}\right) \leq 37 \\ \frac{\tilde{y}_{1}(\tilde{x})-37}{65-37}, & 37 \leq \tilde{y}_{1}\left(\tilde{x}^{q}\right) \leq 65 \\ 1, & \tilde{y}_{1}\left(\tilde{x}^{q}\right) \geq 65\end{cases}
$$


Then, the fuzzy regression, $\tilde{y}_{2}(\tilde{x})$ and $\tilde{s}_{2}\left(\tilde{y}_{2}(\tilde{x})\right)$ for $y_{2}$ are expressed respectively as follows:

$\tilde{y}_{2}(\tilde{x})=(1.18,1.33,1.47)+(3.55,3.92,4.28) \tilde{x}_{1}+(-0.045,-0.044,-0.043) \tilde{x}_{2}+$ $(0.025,0.025,0.025) \tilde{x}_{3}+(0.018,0.019,0.02) \tilde{x}_{4}+(-1.37,-1.18,-0.98) \tilde{x}_{1}^{2}+$ $(-0.18,-0.17,-0.16) \tilde{x}_{1} \tilde{x}_{2}+(0.007,0.008,0.009) \tilde{x}_{2}^{2}$

and

$$
\tilde{s}_{2}\left(\tilde{y}_{2}(\tilde{x})\right)= \begin{cases}1, & \tilde{y}_{2}\left(\tilde{x}^{q}\right) \leq 2.4 \\ \frac{\tilde{y}_{2}(\tilde{x})-4.5}{2.4-4.5}, & 2.4 \leq \tilde{y}_{2}\left(\tilde{x}^{q}\right) \leq 4.5 \\ 0, & \tilde{y}_{2}\left(\tilde{x}^{q}\right) \geq 4.5\end{cases}
$$

Table 6 displays the $\tilde{s}_{r}\left(\tilde{y}_{r}\left(\tilde{x}^{q}\right)\right)$ values for both responses, in which both $\tilde{p}_{1}$ and $\tilde{w}_{1}$ values are equal to $(0.023,0.141,0.26)$, whereas $\tilde{p}_{2}$ and $w_{2}$ are calculated as $(0.243,0.61,0.967)$ and $(0.243,0.551$, $0.783)$, respectively. The $\tilde{s}_{r}\left(\tilde{y}_{r}\left(\tilde{x}^{q}\right)\right)$ values are then used to calculate the fuzzy deviation function, $\tilde{S}_{r}\left(\tilde{y}_{r}\left(\tilde{x}^{q}\right)\right)$, for $y_{1}$ and $y_{2}$ are expressed respectively as:

$$
\begin{aligned}
\tilde{S_{1}}\left(\tilde{y}_{1}\left(\tilde{x}^{q}\right)\right)= & 0.038+0.108 \tilde{x}_{1}+0.342 \tilde{x}_{2}+0.009 \tilde{x}_{3}+0.148 \tilde{x}_{4}+ \\
& 0.031 \tilde{x}_{2} \tilde{x}_{2}+0.002 \tilde{x}_{2} \tilde{x}_{3}+0.006 \tilde{x}_{4} \tilde{x}_{4}
\end{aligned}
$$

and

$$
\begin{gathered}
\tilde{S}_{2}\left(\tilde{y}_{2}\left(\tilde{x}^{q}\right)\right)=0.168+0.427 \tilde{x}_{1}+0.001 \tilde{x}_{2}+0.0005 \tilde{x}_{3}+0.002 \tilde{x}_{4}+ \\
0.225 \tilde{x}_{1}^{2}+0.008 \tilde{x}_{1} \tilde{x}_{2}+0.001 \tilde{x}_{2} \tilde{x}_{2}
\end{gathered}
$$

Table 7 displays the $\tilde{S}_{r}\left(\tilde{y}_{r}\left(\tilde{x}^{q}\right)\right)$ values for both responses, from which the $\tilde{P}_{1}$ and $\tilde{P}_{2}$ values are $(3.89,3.89,3.89)$ and $(0.874 .0 .874$, $0.874)$, respectively, while the $\tilde{W}_{1}$ and $\tilde{W}_{2}$ values are estimated as $(6.717,6.719,6.72)$ and $(0.874 .0 .874,0.874)$, respectively

Table 6. The $\tilde{s}_{r}\left(\tilde{y}_{r}\left(\tilde{x}^{q}\right)\right)$ values of MMR and SR (Case I).

\begin{tabular}{|c|c|c|}
\hline & $\tilde{s}_{1}\left(\tilde{y}_{1}\left(\tilde{x}^{r}\right)\right)$ & $\tilde{s}_{2}\left(\tilde{y}_{2}\left(\tilde{x}^{r}\right)\right)$ \\
\hline$\tilde{x}^{1}$ & $(0.023,0.141,0.26)$ & $(0.32,0.551,0.783)$ \\
\hline$\tilde{x}^{2}$ & $(0.312,0.536,0.761)$ & $(0.243,0.61,0.967)$ \\
\hline
\end{tabular}

Table 7. The $\tilde{S}_{r}\left(\tilde{y}_{r}\left(\tilde{x}^{q}\right)\right)$ values for WEDM process.

\begin{tabular}{|c|c|c|}
\hline & $\tilde{S}_{1}\left(\tilde{y}_{1}\left(\tilde{x}^{q}\right)\right)$ & $\tilde{S}_{2}\left(\tilde{y}_{2}\left(\tilde{x}^{q}\right)\right)$ \\
\hline$\tilde{x}^{1}$ & $(3.89,3.89,3.89)$ & $(0.572,0.572,0.572)$ \\
\hline$\tilde{x}^{2}$ & $(6.717,6.719,6.72)$ & $(0.874,0.887,0.899)$ \\
\hline
\end{tabular}

Finally, the $\tilde{Z}_{r}\left(\tilde{y}_{r}(\tilde{x})\right)$ function is formulated for the two responses as follows:

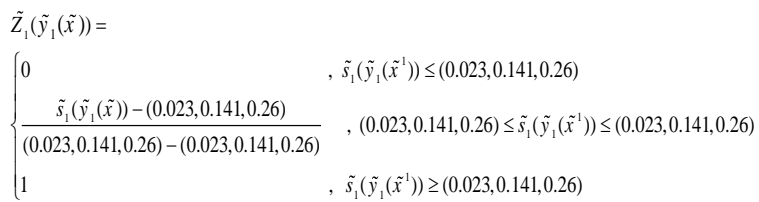

and

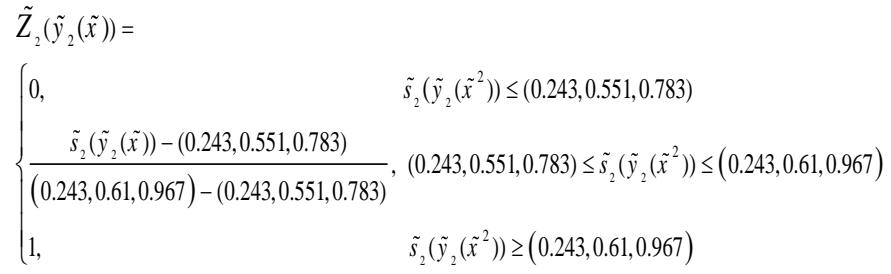

In a similar manner, the $\tilde{T}_{r}\left(\tilde{y}_{r}(\tilde{x})\right)$ functions for $y_{1}$ and $y_{2}$ are written respectively as:

$\tilde{T_{1}}\left(\tilde{y}_{1}(\tilde{x})\right)=$

$\begin{cases}1, & \tilde{S}_{1}\left(\tilde{y}_{1}\left(\tilde{x}^{1}\right)\right) \leq(3.89,3.89,3.89) \\ \frac{(6.717,6.719,6.72)-\tilde{S}_{1}\left(\tilde{y}_{1}(\tilde{x})\right)}{(6.717,6.719,6.72)-(3.89,3.89,3.89)}, & (3.89,3.89,3.89) \leq \tilde{S}_{1}\left(\tilde{y}_{1}\left(\tilde{x}^{1}\right)\right) \leq(6.717,6.719,6.72) \\ 0, & \tilde{S}_{1}\left(\tilde{y}_{1}\left(\tilde{x}^{1}\right)\right) \geq(6.717,6.719,6.72)\end{cases}$

and

$\tilde{T}_{2}\left(\tilde{y}_{2}(\tilde{x})\right)=$
$\left\{\begin{array}{l}1, \quad \tilde{S}_{2}\left(\tilde{y}_{2}\left(\tilde{x}^{2}\right)\right) \leq(0.874,0.887,0.899) \\ \frac{(0.874,0.887,0.899)-\tilde{S}_{2}\left(\tilde{y}_{2}(\tilde{x})\right)}{(0.874,0.887,0.899)-(0.874,0.887,0.899)},(0.874,0.887,0.899) \leq \tilde{S}_{2}\left(\tilde{y}_{2}\left(\tilde{x}^{2}\right)\right) \leq(0.874,0.887,0.899) \\ 0, \quad \tilde{S_{2}\left(\tilde{y}_{2}\left(\tilde{x}^{2}\right)\right) \geq(0.874,0.887,0.899)}\end{array}\right.$

$\tilde{Z}_{r}\left(\tilde{y}_{r}(\tilde{x})\right)= \begin{cases}0, & \tilde{s}_{r}\left(\tilde{y}_{r}\left(\tilde{x}^{r}\right)\right) \leq \tilde{w_{r}} \\ \frac{\tilde{s}_{r}(\tilde{y}}{\left.\left.\tilde{y}_{r}-\tilde{x}\right)\right)-\tilde{L}_{r}}, \quad \tilde{w}_{r} \leq \tilde{s}_{r}\left(\tilde{y}_{r}\left(\tilde{x}^{r}\right)\right) \leq \tilde{p}_{r} \\ 1, \quad \tilde{s}_{r}\left(\tilde{y}_{r}\left(\tilde{x}^{r}\right)\right) \geq \tilde{p}_{r}\end{cases}$

$\tilde{T_{r}}\left(\tilde{y}_{r}(\tilde{x})\right)= \begin{cases}1, & \tilde{S}_{r}\left(\tilde{y}_{r}\left(\tilde{x}^{r}\right)\right) \leq \tilde{P}_{r} \\ \frac{\tilde{W}_{r}-\tilde{S_{r}}\left(\tilde{y}_{r}(\tilde{x})\right)}{\tilde{W}_{r}-\tilde{P}_{r}}, & \tilde{P}_{r} \leq \tilde{S}_{r}\left(\tilde{y}_{r}\left(\tilde{x}^{r}\right)\right) \leq \tilde{W}_{r} \\ 0, & \tilde{S}_{r}\left(\tilde{y}_{r}\left(\tilde{x}^{r}\right)\right) \geq \tilde{W}_{r}\end{cases}$

Finally, the optimization models at the lower, middle and upper; $l$, $m$, and $u$, respectively, were constructed. For example, the optimization model at lower bound of the fuzzy number is expressed as:

$\operatorname{Max} 0.5 \times S^{m}+0.5 \times T^{m}$

s. $t$

$$
\begin{aligned}
-1.418+1.492 x_{1}^{l} & +0.094 x_{2}^{l}-0.043 x_{3}^{l}+0.155 x_{4}^{l} \\
- & 0.017 x_{2}^{l} x_{2}^{l}+0.004 x_{2}^{l} x_{3}^{l}-0.006 x_{4}^{l} x_{4}^{l} \\
- & 0 S^{l} \geq 0.023 \\
1.58-1.69 x_{1}^{l}+ & 0.021 x_{2}^{l}-0.011 x_{3}^{l}-0.008 x_{4}^{l} \\
& +0.653 x_{1}^{l} x_{1}^{l}+0.084 x_{1}^{l} x_{2}^{l} \\
& -0.003 x_{2}^{l} x_{2}^{l}+0 S^{l} \geq 0.243 \\
0.038+0.108 x_{1}^{l} & +0.342 x_{2}^{l}+0.009 x_{3}^{l}+0.148 x_{4}^{l} \\
& +0.031 x_{2}^{l} x_{2}^{l}+0.002 x_{2}^{l} x_{3}^{l} \\
& +0.006 x_{4}^{l} x_{4}^{l}+2.826 T^{l} \leq 6.717
\end{aligned}
$$


$0.168+0.427 x_{1}^{l}+0.001 x_{2}^{l}+0.0005 x_{3}^{l}+0.002 x_{4}^{l}$

$$
\begin{aligned}
& +0.225 x_{1}^{l} x_{1}^{l}+0.008 x_{1}^{l} x_{2}^{l} \\
& +0.001 x_{2}^{l} x_{2}^{l}+0 T^{l} \leq 0.874
\end{aligned}
$$

$0 \leq S^{l} \leq 1$

$0 \leq T^{l} \leq 1$

$X=\left\{x_{1}^{l}, x_{2}^{l}, x_{3}^{l}, x_{4}^{l}\right\} \in[$ Factor Levels $]$

In a similar manner, the optimization models were developed at middle and upper bound of the fuzzy number. The three models were then solved using Lingo 11 Software to determine the values of the fuzzy optimal factor settings. The optimization results showed that be the $l, m$ and $u$ optimal factor settings; Table 8. Experimental design for gasoline process. $x^{* l}=\left(x_{1}^{*}, x_{2}^{*}, x_{3}^{*}, x_{4}^{*}\right), x^{* m}=\left(x_{1}^{*}, x_{2}^{*}, x_{3}^{*}, x_{4}^{*}\right)$, and $x^{* u}=\left(x_{1}^{*}, x_{2}^{*}, x_{3}^{*}, x_{4}^{*}\right)$, respectively, are $(0.6,4.33,8,8),(0.6,4,8,8)$ and $(0.60,4,8,8)$. At these fuzzy optimal factor settings, the calculated $\tilde{y}_{1}$ and $\tilde{y}_{2}$ values were $(55.72,57.73,60.16)$ and $(2.65,3.16,3.64)$, respectively.

\section{Case study II: Gasoline Production Process}

Bashiri et al. [22] used the regression and artificial neural network (ANN) approaches to enhance the vapor pressure $\left(y_{1}, \mathrm{RVP}, \mathrm{LTB}\right)$, rate of octane number $\left(y_{2}, \mathrm{RON}, \mathrm{STB}\right)$ and density $\left(y_{3}, \mathrm{DEN}, \mathrm{LTB}\right)$ in a gasoline production process. Five controllable process factors

\begin{tabular}{|c|c|c|c|c|c|c|c|c|c|c|c|}
\hline \multirow{3}{*}{$\begin{array}{c}\text { Ex. } \\
i \\
1 \\
\end{array}$} & \multicolumn{5}{|c|}{ Control factors } & \multicolumn{6}{|c|}{ Responses (2 replicates) } \\
\hline & $x_{1}$ & $x_{2}$ & $x_{3}$ & $x_{4}$ & $x_{5}$ & \multicolumn{2}{|c|}{$y_{11 i} \quad y_{12 i}$} & \multicolumn{2}{|c|}{$y_{21 i} \quad y_{22 i}$} & \multicolumn{2}{|c|}{$y_{31 i} \quad y_{32 i}$} \\
\hline & 3.5 & 5 & 3 & 3 & 5 & 64 & 63 & 89 & 88.3 & 0.751 & 0.751 \\
\hline 2 & 5 & 10 & 3 & 3 & 5 & 63 & 62.5 & 93.5 & 92.4 & 0.755 & 0.755 \\
\hline 3 & 3.5 & 5 & 5 & 5 & 5 & 62 & 60.5 & 88 & 87.1 & 0.753 & 0.754 \\
\hline 4 & 5 & 10 & 5 & 5 & 5 & 61.5 & 60.5 & 94 & 93 & 0.756 & 0.756 \\
\hline 5 & 5 & 5 & 5 & 3 & 7.5 & 63 & 62 & 93.2 & 92.1 & 0.759 & 0.759 \\
\hline 6 & 3.5 & 10 & 5 & 3 & 7.5 & 62.5 & 61.5 & 91.8 & 91 & 0.756 & 0.756 \\
\hline 7 & 5 & 5 & 3 & 5 & 7.5 & 62 & 61 & 93.5 & 92.4 & 0.758 & 0.759 \\
\hline 8 & 3.5 & 10 & 3 & 5 & 7.5 & 61 & 59.5 & 91.5 & 90.5 & 0.757 & 0.757 \\
\hline
\end{tabular}
were studied utilizing of the $L_{8}$ array shown in Table 8 .
The proposed procedure starts by adopting the GA technique to determine the optimal factor settings for each response replicate. The obtained optimal level values are shown in Table 9. Utilizing these values, the fitted fuzzy regression models $\tilde{y}_{1}(\tilde{x}), \tilde{y}_{2}(\tilde{x})$, and $\tilde{y}_{3}(\tilde{x})$ are constructed for responses $y_{1}$, and $y_{2}$, and $y_{3}$, respectively. For example, the $\tilde{y}_{1}(\tilde{x})$ is represented as:

$$
\begin{aligned}
& \tilde{y}_{1}(\tilde{x}) \\
& =(70.28583,70.4165,70.54717)+ \\
& (0.106727,0.29165,0.476573) x_{1} \\
& +(-0.43864,-0.3875,-0.33636) x_{2}+ \\
& (-0.78271,-0.625,-0.46729) x_{3}+ \\
& (-0.83157,-0.75,-0.66843) x_{4}+ \\
& (-0.63158,-0.525,-0.41842) x_{5}+ \\
& (0.007387,0.01,0.012613) x_{2} x_{3} x_{5}
\end{aligned}
$$

Then, the fuzzy desirability functions; $\tilde{s}_{1}\left(\tilde{y}_{1}\left(\tilde{x}^{q}\right)\right), \tilde{s}_{2}\left(\tilde{y}_{2}\left(\tilde{x}^{q}\right)\right)$, and $\tilde{s}_{3}\left(\tilde{y}_{3}\left(\tilde{x}^{q}\right)\right)$ for $\tilde{y}_{1}(\tilde{x}), \tilde{y}_{2}(\tilde{x})$, and $\tilde{y}_{3}(\tilde{x})$ are developed respectively as follows:

$$
\begin{gathered}
\tilde{s}_{1}\left(\tilde{y}_{1}(\tilde{x})\right)= \begin{cases}0, & \tilde{y}_{1}\left(\tilde{x}^{r}\right) \leq 57 \\
66-57 & 57 \leq \tilde{y}_{1}\left(\tilde{x}^{r}\right) \leq 66 \\
1, & \tilde{y}_{1}\left(\tilde{x}^{r}\right) \geq 66\end{cases} \\
\tilde{s}_{2}\left(\tilde{y}_{2}(\tilde{x})\right)= \begin{cases}1, & \tilde{y}_{2}\left(\tilde{x}^{r}\right) \leq 85 \\
\frac{\tilde{y}_{2}(\tilde{x})-98}{85-98}, & 85 \leq \tilde{y}_{2}\left(\tilde{x}^{r}\right) \leq 98 \\
0, & \tilde{y}_{2}\left(\tilde{x}^{r}\right) \geq 98\end{cases} \\
\tilde{s}_{3}\left(\tilde{y}_{3}(\tilde{x})\right)= \begin{cases}0, & \tilde{y}_{3}\left(\tilde{x}^{r}\right) \leq 0.75 \\
\frac{\tilde{y}_{3}(\tilde{x})-0.75}{0.78-0.75}, & 0.75 \leq \tilde{y}_{3}\left(\tilde{x}^{r}\right) \leq 0.78 \\
1, & \tilde{y}_{3}\left(\tilde{x}^{r}\right) \geq 0.78\end{cases}
\end{gathered}
$$

The values of $\tilde{s}_{r}\left(\tilde{y}_{r}\left(\tilde{x}^{q}\right)\right)$ are then calculated and then listed in Table 10. These values are employed to calculate the $\tilde{S}_{r}\left(\tilde{y}_{r}\left(\tilde{x}^{q}\right)\right)$ values displayed in Table 11. The values of $x^{* l}=\left(x_{1}^{*}, x_{2}^{*}, x_{3}^{*}, x_{4}^{*}, x_{5}^{*}\right)$ are found $(5,5,3,3.7 .5)$. Similarly, the $x^{* m}=\left(x_{1}^{*}, x_{2}^{*}, x_{3}^{*}, x_{4}^{*}, x_{5}^{*}\right)$ and $x^{* u}=\left(x_{1}^{*}, x_{2}^{*}, x_{3}^{*}, x_{4}^{*}, x_{5}^{*}\right)$ values are found to be $(5,5,3,3.8,7.5)$ and $(4.74,5,5,3.8,7.5)$, respectively. At these fuzzy optimal factor settings, the corresponding response values of $\tilde{y}_{1}, \tilde{y}_{2}$ and $\tilde{y}_{3}$ are calculated as $(59.877,62.379,65.476)$, $(91.39,93.17,94.07)$ and $(0.757,0.7578,0.7593)$, respectively.

Table 9. The GA optimal factor setting for gasoline process.

\begin{tabular}{|c|c|c|c|c|c|c|}
\hline Factor & $\boldsymbol{y}_{\mathbf{1 1}}$ & $\boldsymbol{y}_{\mathbf{1 2}}$ & $\boldsymbol{y}_{\mathbf{2 1}}$ & $\boldsymbol{y}_{\mathbf{2 2}}$ & $\boldsymbol{y}_{\mathbf{3 1}}$ & $\boldsymbol{y}_{\mathbf{3 2}}$ \\
\hline$x_{1}{ }^{*}$ & 4.22 & 3.50 & 5.00 & 5.00 & 5.00 & 5.00 \\
\hline$x_{2}{ }^{*}$ & 10.00 & 5.04 & 10.00 & 10.00 & 9.94 & 5.00 \\
\hline$x_{3}{ }^{*}$ & 3.68 & 5.00 & 4.99 & 4.97 & 4.99 & 4.98 \\
\hline$x_{4}{ }^{*}$ & 5.00 & 5.00 & 3.13 & 3.00 & 5.00 & 5.00 \\
\hline$x_{5}{ }^{*}$ & 7.39 & 7.48 & 7.50 & 7.50 & 7.50 & 7.49 \\
\hline
\end{tabular}

Table 10. The $\tilde{s}_{r}\left(\tilde{y}_{r}\left(\tilde{x}^{q}\right)\right)$ values for gasoline process.

\begin{tabular}{|c|c|c|c|}
\hline & $\tilde{s}_{1}\left(\tilde{y}_{1}\left(\tilde{x}^{q}\right)\right)$ & $\tilde{s}_{2}\left(\tilde{y}_{2}\left(\tilde{x}^{q}\right)\right)$ & $\tilde{s}_{3}\left(\tilde{y}_{3}\left(\tilde{x}^{q}\right)\right)$ \\
\hline$\tilde{x}^{1}$ & $(0.13,0.41,0.94)$ & $(0.08,0.54,0.83)$ & $(0.16,0.24,0.31)$ \\
\hline$\tilde{x}^{2}$ & $(0.12,0.60,1.0)$ & $(0.02,0.24,0.46)$ & $(0.27,0.30,0.32)$ \\
\hline$\tilde{x}^{3}$ & $(0.04,0.44,0.96)$ & $(0,0.34,0.63)$ & $(0.30,0.32,0.35)$ \\
\hline
\end{tabular}

Table 11. The $\tilde{S}_{r}\left(\tilde{y}_{r}\left(\tilde{x}^{q}\right)\right)$ values for gasoline process.

\begin{tabular}{|c|c|c|c|}
\hline & $\tilde{S}_{1}\left(\tilde{y}_{1}\left(\tilde{x}^{q}\right)\right)$ & $\tilde{S}_{2}\left(\tilde{y}_{2}\left(\tilde{x}^{q}\right)\right)$ & $\tilde{S}_{1}\left(\tilde{y}_{1}\left(\tilde{x}^{q}\right)\right)$ \\
\hline$\tilde{x}^{1}$ & $(13.61,15.95$, & $(8.53,11.36,14.44)$ & $(0.0022,0.0028$, \\
& $18.34)$ & $0.0034)$ \\
\hline$\tilde{x}^{2}$ & $(17.61,17.66$, & $(14.16,14.18,14.2)$ & $(0.0034,0.0034$, \\
& $17.71)$ & $0.0034)$ \\
\hline$\tilde{x}^{3}$ & $\begin{array}{c}(16.51,17.64, \\
18.77)\end{array}$ & $(10.466,12.74$, & $(0.0029,0.0032$, \\
& \multicolumn{2}{|c}{$15.02)$} & $0.0035)$ \\
\hline
\end{tabular}

\section{Case study III: Sputtering Process}

Chen et al. [43] used the Taguchi-grey relational method optimize 
parameters of the sputtering process for three quality responses; deposition rate ( $\left.y_{1}, \mathrm{LTB}, \mathrm{DR}\right)$, electrical resistively ( $\left.y_{2}, \mathrm{STB}, \mathrm{ER}\right)$, and optical transmittance ( $\left.y_{3}, \mathrm{LTB}, \mathrm{OT}\right)$. Five controllable process factors; $x_{1}, \ldots, x_{5}$, were investigated via the $L_{18}$ array shown in
Table 12.

Table 12. Experimental data for the sputtering process.

\begin{tabular}{|c|c|c|c|c|c|c|c|c|c|c|c|}
\hline \multirow{2}{*}{ Ex. $i$} & \multicolumn{5}{|c|}{ Factor } & \multicolumn{2}{|c|}{ DR } & \multicolumn{2}{|c|}{ ER } & \multicolumn{2}{|c|}{ OT } \\
\hline & $x_{1}$ & $x_{2}$ & $x_{3}$ & $x_{4}$ & $x_{5}$ & $y_{i 11}$ & $y_{i 12}$ & $y_{i 21}$ & $y_{i 22}$ & $y_{i 31}$ & $y_{i 32}$ \\
\hline 1 & 50 & 0.13 & 30 & 25 & 0 & 4.5 & 4.7 & 14.9 & 15.3 & 88.4 & 88.4 \\
\hline 2 & 50 & 0.67 & 60 & 50 & 100 & 5.6 & 5.6 & 9.8 & 9.7 & 87.7 & 87.7 \\
\hline 3 & 50 & 1.33 & 90 & 100 & 200 & 5.0 & 4.9 & 7.9 & 7.8 & 88.1 & 88.1 \\
\hline 4 & 100 & 0.13 & 30 & 50 & 100 & 9.6 & 9.3 & 5.4 & 5.6 & 89.2 & 89.3 \\
\hline 5 & 100 & 0.67 & 60 & 100 & 200 & 11.1 & 11.3 & 4.6 & 4.3 & 87.1 & 87.0 \\
\hline 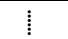 & 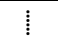 & 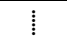 & 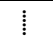 & 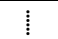 & 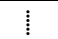 & 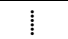 & $\vdots$ & 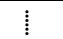 & $\vdots$ & 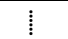 & 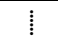 \\
\hline 15 & 100 & 1.33 & 30 & 50 & 200 & 10.7 & 10.8 & 5.5 & 5.7 & 88.4 & 88.3 \\
\hline 16 & 200 & 0.13 & 90 & 50 & 200 & 19.5 & 19.4 & 1.0 & 1.1 & 83.1 & 83.1 \\
\hline 17 & 200 & 0.67 & 30 & 100 & 0 & 22.1 & 22.0 & 1.2 & 1.3 & 85.7 & 85.7 \\
\hline 18 & 200 & 1.33 & 60 & 25 & 100 & 20.5 & 20.5 & 1.4 & 1.3 & 83.9 & 83.7 \\
\hline
\end{tabular}

The regression models were developed for all response replicates. For illustration, the regression models for $y_{11}$ is represented by:

$y_{11}(x)=-1.94+0.12 x_{1}+1.24 x_{2}+0.02 x_{3}-0.01 x_{4}+$

$0.01 x_{5}-0.003 x_{1} x_{2}-0.0001 x_{1} x_{3}+0.0001 x_{1} x_{4}-$

$0.0001 x_{1} x_{5}-0.014 x_{2} x_{3}-0.000004 x_{5}^{2}-$

$0.000004 x_{2} x_{3} x_{4} \quad R^{2}{ }_{\text {adj }}=98.81 \%$

While, the regression models for $y_{12}$ is written as:

$$
\begin{aligned}
y_{12}(x)=-1.86+ & 0.12 x_{1}+0.98 x_{2}+0.04 x_{3}-0.02 x_{4} \\
& +0.01 x_{5}+0.0001 x_{1} x_{2}-0.0002 x_{1} x_{3} \\
& +0.0001 x_{1} x_{4}-0.0001 x_{1} x_{5}-0.02 x_{2} x_{3} \\
& +0.00002 x_{5}^{2} \\
& +0.00003 x_{2} x_{3} x_{4} \quad R_{\text {adj }}^{2}=97.93 \%
\end{aligned}
$$

The acceptable ranges of the controllable factors are decided as follows:

$$
50 \leq x_{1} \leq 200,0.33 \leq x_{2} \leq 1.33,{ }_{30} \leq x_{3} \leq 90,25 \leq x_{4} \leq 100,0 \leq x_{5} \leq 200
$$

The optimal settings of process factors were obtained by GA technique and then displayed in Table 13.

Table 13. GA optimal factor settings for sputtering process.

\begin{tabular}{|c|c|c|c|c|c|c|}
\hline fact & $y_{11}$ & $y_{12}$ & $y_{21}$ & $y_{22}$ & $y_{31}$ & $y_{32}$ \\
\hline$x_{1}{ }^{*}$ & 50.00 & 50.00 & 199.68 & 199.92 & 99.98 & 99.99 \\
\hline$x_{2}{ }^{*}$ & 1.33 & 1.33 & 0.34 & 1.33 & 1.33 & 1.33 \\
\hline$x_{3}{ }^{*}$ & 89.93 & 30.00 & 43.62 & 89.98 & 89.99 & 89.88 \\
\hline$x_{4}{ }^{*}$ & 25.00 & 25.00 & 94.75 & 25.00 & 75.77 & 61.83 \\
\hline$x_{5}{ }^{*}$ & 0.001 & 0.14 & 70.28 & 28.28 & 4.85 & 11.88 \\
\hline
\end{tabular}

Utilizing the results in Table 13, the fuzzy regression for $\tilde{y}_{1}(\tilde{x}), \tilde{y}_{2}(\tilde{x})$, and $\tilde{y}_{3}(\tilde{x})$ were developed for the three responses. For illustration, the regression model for $\tilde{y}_{1}(\tilde{x})$ is written as:

$\tilde{y}_{1}(\tilde{x})=(-1.95,-1.9,-1.85)+(0.12,0.12,0.12) \mathrm{x}_{1}+$

$(0.96,1.11,1.26) \mathrm{x}_{2}+(0.02,0.03,0.04) \mathrm{x}_{3}+$

$(-0.02,-0.017,-0.012) \mathrm{x}_{4}+(0.01,0.01,0.01)$

$\mathrm{x}_{5}+(-0.003,-0.001,0.0003) x_{1} x_{2}+$

$(-0.0002,-0.0001,-0.0001) x_{1} x_{3}+$

$(0.0001,0.0001,0.0001) x_{1} x_{4}+$

$(-0.0001,-0.0001,-0.0001) x_{1} x_{5}+(-0.02,-0.02,-0.01) x_{2} x_{3}+$
$(-.00001,0.00001,0.00003) x_{2} x_{3} x_{4}+$ $(-0.00001,0.00001,0.00002) x_{5}^{2}$

Then, the fuzzy desirability functions, $\tilde{s}_{r}\left(\tilde{y}_{r}\left(\tilde{x}^{q}\right)\right)$, are constructed for all quality responses. The $\tilde{s}_{1}\left(\tilde{y}_{1}\left(\tilde{x}^{q}\right)\right)$, for example, is developed as:

$\tilde{s}_{1}\left(\tilde{y}_{1}\left(\tilde{x}^{q}\right)\right)= \begin{cases}0, & \tilde{y}_{1}\left(\tilde{x}^{q}\right) \leq 4 \\ \frac{\tilde{y}_{1}(\tilde{x})-4}{24-4}, & 4 \leq \tilde{y}_{1}\left(\tilde{x}^{q}\right) \leq 24 \\ 1, & \tilde{y}_{1}\left(\tilde{x}^{q}\right) \geq 24\end{cases}$

The corresponding $\tilde{Z}_{1}\left(\tilde{y}_{1}(\tilde{x})\right)$ and $\tilde{T}_{j}\left(\tilde{y}_{1}(\tilde{x})\right)$ are then formulated as follows:

$\tilde{Z}_{1}\left(\tilde{y}_{1}(\tilde{x})\right.$

$\int 0, \quad \tilde{s}_{1}\left(\tilde{y}_{1}\left(\tilde{x}^{q}\right)\right) \leq(0.013,0.054,0.15)$

$=\left\{\begin{array}{l}\frac{\tilde{s}_{1}\left(\tilde{y}_{1}(\tilde{x})\right)-(0.013,0.054,0.15)}{(0.013,0.054,0.15)-(0.013,0.054,0.15)},(0.013,0.054,0.15) \leq \tilde{s}_{1}\left(\tilde{y}_{1}\left(\tilde{x}^{q}\right)\right) \leq(0.013,0.054,0.15)\end{array}\right.$

$1, \quad \tilde{s}_{1}\left(\tilde{y}_{1}\left(\tilde{x}^{q}\right)\right) \geq(0.013,0.054,0.15)$

$\tilde{T_{1}}\left(\tilde{y}_{1}(\tilde{x})\right.$

$= \begin{cases}1, & \tilde{S}_{1}\left(\tilde{y}_{1}\left(\tilde{x}^{q}\right)\right) \leq(3.68,6.59,9.49) \\ \frac{(11.71,12.22,21.15)-\tilde{S}_{1}\left(\tilde{y}_{1}\left(\tilde{x}_{)}\right)\right)}{(11.71,12.22,21.15)-(3.68,6.58,9.49)}, & (3.68,6.58,9.49) \leq \tilde{S}_{1}\left(\tilde{y}_{1}\left(\tilde{x}^{q}\right)\right) \leq(11.71,12.22,21.15) \\ 0, & , \tilde{S}_{1}\left(\tilde{y}_{1}\left(\tilde{x}^{q}\right)\right) \geq(11.71,12.22,21.15)\end{cases}$

The $\tilde{Z}_{j}(x)$ and $\tilde{T}_{j}(x)$ functions of the other responses are formulated in a similar manner.

Finally, the optimization models constructed at the $l, m$, and $u$ bounds and then solved to obtain the fuzzy optimal settings of process factors shown in Table 14.

Table 13. The fuzzy optimal factor settings for sputtering process.

\begin{tabular}{|c|c|c|c|}
\hline Factor & Model $l$ & Model $m$ & Model $u$ \\
\hline$x_{1}{ }^{*}$ & 198.29 & 198.906 & 59.743 \\
\hline$x_{2}{ }^{*}$ & 0.13 & 0.618 & 1.33 \\
\hline$x_{3}{ }^{*}$ & 30.00 & 30.00 & 30.00 \\
\hline$x_{4}{ }^{*}$ & 25.00 & 43.35 & 25.00 \\
\hline$x_{5}{ }^{*}$ & 0.00 & 29.336 & 0.00 \\
\hline
\end{tabular}

Substituting the fuzzy optimal settings shown in Table 13, the estimated values of $\tilde{y}_{1}, \tilde{y}_{2}$ and $\tilde{y}_{3}$ are $(7.074,20.54,21.34),(1.27$, $1.51,9.62)$ and $(87.42,88.28,88.53)$, respectively. 


\section{Results and Discussion}

A comparison between the optimization results obtained using the GA-fuzzy procedure and those adopted on the studied case studies is conducted as follows:

- For the WEDM process [5] as shown in Fig. 2, the initial MRR and SR values were 52.15 and 3.43 , respectively. Using the Taguchi method (GA-Fuzzy), the values of MRR and SR values at the optimal factor settings are $68.56(55.72,57.73,60.16)$ and 3.45 $(2.65,3.16,3.64)$, respectively. It is noticed that the Taguchi method provides the largest improvement in MRR, whereas the GA-fuzzy procedure resulted in the largest improvement in SR. However, the Taguchi method does not rely on mathematical relation between process factors and each response. Moreover, it ignores preferences on product and process settings, and lacks the ability to deal with uncertainty due to measurement and process variations.

- For the gasoline production process [22] as shown in Fig. 3, using the ANN technique (GA-fuzzy procedure) the obtained RVP (LTB), RON (STB) and DEN (LTB) values are calculated as 58.99 (59.87, 62.37, 65.477), 93.96 (91.39, 93.17, 94.07), and 0.76 $(0.757,0.7578,0.7593)$, respectively. It is clear that the GA-fuzzy provide the largest improvements in RVP and RON, respectively. While, both approaches provide almost the same improvement in DEN. Although the ANN is widely used, it has a number of limitations, such as, its "black box" nature and limited ability to explicitly determine possible causal relationships, large computational burden, and proneness to overfitting. Moreover, the ANN requires sufficient data set for training and validation. Finally, the used ANN approach ignored the fuzziness nature in quality characteristics and process settings.

- For the sputtering process [43] as shown in Fig. 4, it is noticed that the DR (LTB), ER (STB) and OT (LTB) values at initial factor settings are $21.81,1.61$ and 85.73 , respectively. The corresponding values at the optimal settings by using the Grey-Taguchi method are $22.19,1.25$ and 86.85 , respectively. Using the GA-Fuzzy procedure, the DR, ER, and OT values range from 7.074 to 21.34 , 1.27 to 9.62 , and 87.42 to 88.3 , respectively. It is seen that the greyTaguchi method provides slightly larger improvements in the three quality characteristics. Using the GA-fuzzy approach the $\mathrm{u}, \mathrm{l}$, and $\mathrm{u}$ values of DR $(=21.34)$, ER $(=1.27)$, and OT $(=88.3)$, respectively, are slightly differ from those obtained by the Taguchi method and the grey relational approach. However, the grey relational analysis is based on ranking rather than mathematical modeling and thus it may not provide optimal process settings. Besides, Taguchi method and grey relational approach failed in handling fuzziness of the quality characteristics, which is due to measurement and process variations. For illustration, using the GA-fuzzy procedure the DR values at the $\mathrm{u}(=21.34)$ and 1 (= 7.074) levels differ significantly because of the existence of variations, which reveals the effectiveness of this approach in handling uncertainty.
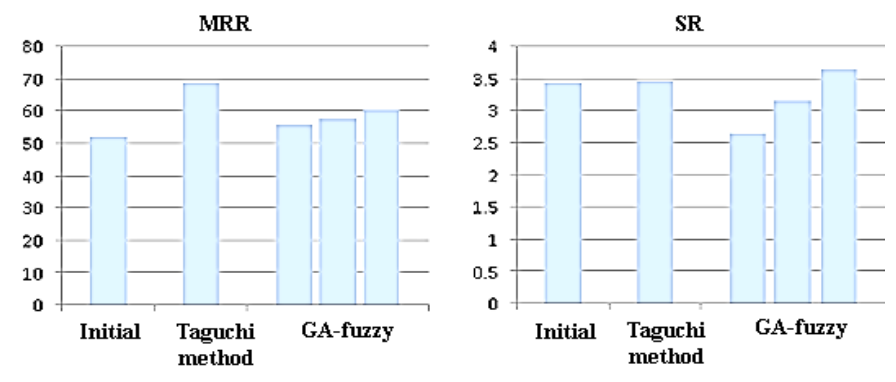

Fig. 2. Results comparison for WEDM process.

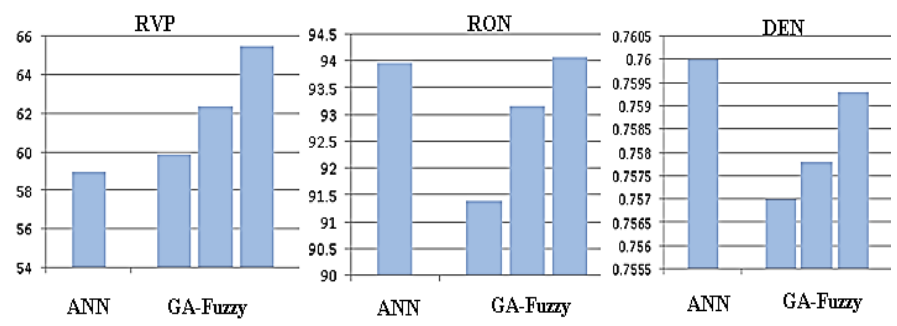

Fig. 3. Results comparison for gasoline process.
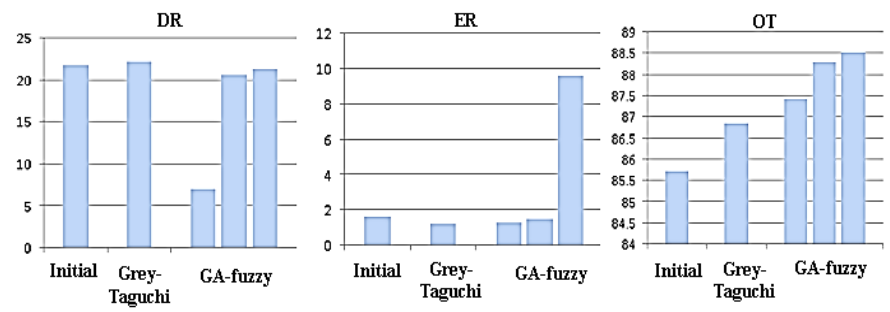

Fig. 4. Results comparison for sputtering process.

In summary, the proposed GA-fuzzy procedure has several benefits, including:

i. It effectively deals with uncertainty due to fuzzy quality characteristics and process settings by providing fuzzy rather than crisp optimal factor settings.

ii. It is relies on mathematical models to depict the relationships between the quality characteristic and process factors. This enables process engineers evaluate and predict accurately values of quality characteristic and process performance under fuzziness.

iii. It handles uncertainty/variations in the observations between the replicates of each response, which improves accuracy in determining the optimal factor settings.

iv. It conducts two-stage optimization. In the first stage, it uses the genetic algorithm technique, which is found effective providing optimal/near optimal solutions, to determine the crisp optimal factor settings for each response. Then, it adopts fuzzy goal programming to handle fuzziness in responses and process settings and then optimizing performance for multiple responses.

Nevertheless, the GA-fuzzy approach requires a moderate knowledge and proficiency in statistics and computer skills. In addition, process engineers should possess extensive understanding of experimental design and analysis, regression modelling and optimization. This may increase the complexity of this approach. 


\section{Conclusions}

This research developed a GA-fuzzy procedure for optimizing a manufacturing process for multiple characteristics under uncertainty. The GA technique was initially adopted to optimize factor levels for each response's replicate separately, which were then employed to formulate a fuzzy regression for each response. The fuzzy desirability function and deviation functions were developed and finally utilized in constructing the fuzzy optimization model. Three case studies in manufacturing were used for illustration. In contrast to previously used approaches, results showed that the proposed procedure has efficiently optimized process settings under measurement and process variations. In conclusions, the proposed procedure provides valuable information to process engineers about the relationships between quality characteristics and process factors and the impact of uncertainty on product and process performance. Such feedback can support them in taking proper corrective and preventive actions. Future research considers combining neural networks and fuzzy goal programming to optimize process performance under uncertainty.

\section{References}

[1] G. Taguchi, "Taguchi Methods. Research and Development. Vol. 1," Dearborn, MI, American Suppliers Institute Press, 1991.

[2] N. M. Mehat and S. Kamaruddin, "Investigating the effects of injection molding parameters on the mechanical properties of recycled plastic parts using the Taguchi method," Materials and Manufacturing Processes, vol. 26, no. 2, pp. 202-209, 2011.

[3] R. Çakıroğlu, A. Acır, "Optimization of cutting parameters on drill bit temperature in drilling by Taguchi method," Measurement, vol. 46, pp. 3525-3531, 2013.

[4] B.V. Dharmendra, S. P. Kodali, B. N. Rao, "A simple and reliable Taguchi approach for multi-objective optimization to identify optimal process parameters in nano-powder-mixed electrical discharge machining of INCONEL800 with copper electrode," Heliyon, vol. 5, no. 8, pp. e02326, 2019.

[5] https://doi.org/10.1016/j.heliyon.2019.e02326.

[6] R. Ramakrishnan and L. Karunamoorthy, "Modeling and multiresponse Optimization of Inconel 718 on machining of CNC WEDM process," Journal of Materials Processing Technology, vol. 207, pp. 343-349, 2008.

[7] S. Athreya and Y.D. Venkatesh, "Application of Taguchi method for optimization of process parameters in improving the surface roughness of lathe facing operation," International Refereed Journal of Engineering and Science (IRJES), vol. 1, no. 3, pp. 13-19, 2012.

[8] J. Antony, R.B. Anand, M. Kumar and M.K., "Tiwari Multiple response optimization using Taguchi methodology and neuro-fuzzy based model," Journal of Manufacturing Technology Management, vol. 17, pp. 908-925, 2006.

[9] H. Singh and P. Kumar," Optimizing multi-machining characteristics through Taguchi's approach and utility concept," Journal of Manufacturing Technology, vol. 17, no. 2, pp. 255-274, 2006.

[10] A. Mishra and A. Gangeleb, "Multi-Objective Optimization in Turning of Cylindrical Bars of AISI 1045 Steel through Taguchi's Method and Utility concept," International Journal of Sciences: Basic and Applied Research (IJSBAR), vol.12, pp. 28-36, 2013.

[11]R. Jeyapaul, P. Shahabudeen and K. Krishnaiah, "Simultaneous optimization of multi-response problems in the Taguchi method using genetic algorithm," International Journal of Advanced Manufacturing Technology, vol. 30, pp. 870-878, 2006.

[12] A.R. Yıldız, N. Öztürk, N. Kaya, and F. Öztürk, "Hybrid multiobjective shape design optimization using Taguchi's method and genetic algorithm," Struct Multidisc Optim, vol. 34, pp. 317-332, 2006.

[13] A. Anju and_S. Rajender, "A Paper on Multiple Objective Functions of Genetic Algorithm," International Journal of Computer Applications, vol.119, no.10, pp. 0975 -8887, 2015.

[14] K-T., Chiang. "The optimal process conditions of an injection-molded thermoplastic part with a thin shell feature using grey-fuzzy logic: a case study on machining the PC/ABS cell phone shell" Mater Des., vol. 28, pp. 1851-1860, 2007.

[15] A. Aman, S. Hari, K. Pradeep, and S., "Manmohan Optimizing power consumption for CNC turned parts using response surface methodology and Taguchi's technique-A comparative analysis, " Journal of materials processing technology, pp. 373-384, 2008.

[16]A. Al-Refaie, "Optimizing SMT performance using comparisons of efficiency between different systems technique in DEA," IEEE Transactions on Electronics Packaging Manufacturing; vol. 32, pp. 256-264, 2009.

[17] Y. L. Chen, L. H. Chen, and C. Y. Huang, "Fuzzy goal programming approach to solve the equipment-purchasing problem of an FMC," International Journal of Industrial Engineering: Theory, Applications and Practice, vol.16, no. 4, pp. 270-281, 2009.

[18]_Y. Kazancoglu, U. Esme, M. Bayramoglu, O.Guven, and S. Oz gun, " Multi-objective optimization of the cutting forces in turning operations using the grey-based Taguchi method," Materials and Technology, vol. 45, pp. 105-110, 2011.

[19] S. Lal, S. Kumar, Z. Khan, "Multiresponse optimization of wire electrical discharge machining process parameters for Al7075/A12O3/SiC hybrid composite using Taguchi-based grey relational analysis," Proc IMechE, Part B: J Engineering Manufacture, vol. 229: pp. 229-237, 2015.

[20]D.V. Kumar, P.S. Kumar, B. Kumaragurubharan, T. Senthilkumar," Experimental investigation of process parameters in EDM for INCOLOY600 using Taguchi-GRA," Int. J. Eng. Sci. Comp., vol. 6, pp. 6206-6206, 2016.

[21]H-C. Lin, C-T. Su, C-C. Wang, "Parameter optimization of continuous sputtering process based on Taguchi methods, neural networks, desirability function, and genetic algorithms. Expert Syst Appl., vol. 39: pp. 12918-12925, 2012.

[22]G. Xu, Z. Yang, G. Long, "Multi-objective optimization of MIMO plastic injection molding process conditions based on particle swarm optimization," Int J Adv Manuf Technol, vol. 58, pp. 521-531, 2013.

[23]M. Bashiri, H. R.Rezaei, A. F. Geranmayeh, \& F. Ghobadi, "A comparison of regression and neural network based for multiple response optimization in a real case study of gasoline production process," Journal of Industrial and Systems Engineering, vol. 8, no. 3, pp. 77-94, 2015.

[24]S. Karabulut, "Optimization of surface roughness and cutting force during AA7039/A12O3 metal matrix composites milling using neural networks and Taguchi method," Measurement, vol. 66, pp. 139-149, 2015.

[25]C. Venkatesh, R. Venkatesan, "Optimization of process parameters of hot extrusion of SiC/Al 6061 composite using Taguchi's technique and upper bound technique," Mater Manuf. 
Process, vol. 30, pp. 85-92, 2015.

[26]A. Al-Refaie, T. Chen, R. Al-Athamneh, "Fuzzy neural network approach to optimizing process performance by using multiple responses," Journal of Ambient Intelligent Humanized Computing, vol.7, pp. 801-816, 2016.

[27]A. Al-Refaie, "Optimal performance of plastic pipes' extrusion process using Min-Max model in fuzzy goal programming, " Proceedings of the Institution of Mechanical Engineers, Part E: Journal of Process Mechanical Engineering, vol. 231, no. 4, pp. 888-898, 2017.

[28]A. Al-Refaie, G. Bani Domi , R. Abdullah, "A fuzzy goal programming-regression approach to optimize process performance of multiple responses under uncertainty," International Journal of Management Science and Engineering Management, vol. 14, no. 1, pp. 20-32, 2019. doi: 10.1080/17509653.2018.1467802.

[29] A. Al-Refaie, N. Lepkova, G. Abbasi, G. Bani Domi, "Optimization of process performance by multiple pentagon fuzzy responses: Case studies of wire-electrical discharge machining and sputtering process," Advances in Production Engineering \& Management, vol. 15, no. 3, pp 307-317, 2020.

[30]https://doi.org/10.14743/apem2020.3.367

[31] U. Lee, S. Park, I. Lee, "Robust design optimization (RDO) of thermoelectric generator system using non-dominated sorting genetic algorithm II (NSGA-II)," Energy, Vol. 196, pp. 117090, 2020.

[32]J. H., "Holland Adaptation in natural and artificial systems," Ann Arbor: University of Michigan Press; 1975

[33]D.E. Goldberg, "Genetic Algorithms in Search, Optimization, and Machine Learning," Addison-Wesley Professional: Boston, MA, USA, 1989.

[34]X. Wu, C-H. Chu, Y. Wang, W. Yan, "A genetic algorithm for cellular manufacturing design and layout," European J Oper Res., vol. 181, no. 1, pp.156-167, 2007.

[35] S. N. Sivanandam and S. N. Deepa, "Introduction to Genetic Algorithms," Springer, (1 ${ }^{\text {st }}$ d), Berlin, Germany, 2008.

[36]C.K.H. Lee, "A review of applications of genetic algorithms in operations management," Eng. Appl. Artif. Intell., vol. 76, pp. 1-12, 2018.

[37]S. Katoch, S.S. Chauhan, \& V. Kumar, "A review on genetic algorithm: past, present, and future," Multimed Tools Appl., vol. 80, pp. 8091-8126, 2021. https://doi.org/10.1007/s11042-020-101396

[38]Z. Liu, A. Liu, C., " Wang and Z. Niu, "Evolving neural network using real coded genetic algorithm (GA) for multispectral image classification", Future Generation Computer Systems, vol. 20, no. 7, pp. 1119-1129, 2004.

[39]G. Candan, H. Yazgan, "Genetic algorithm parameter optimization using Taguchi method for a flexible manufacturing system scheduling problem," Int J Prod Res., vol. 53, pp. 897-915, 2015.

[40] M. A. Şahman, M. Çunkaş, Ş. İnal, F. İnal, B. Coşkun \& U. Taşkiran, "Cost optimization of feed mixes by genetic algorithms. Advances in Engineering Software, vol. 40, no. 10, pp. 965-974, 2009.

[41]R. Zhang, S.K. Ong, A.Y. C. Nee, "A simulation-based genetic algorithm approach for remanufacturing process planning and scheduling," Appl Soft Comput vol. 37, pp. 521-532, 2015.

[42] Y-B Park, J-S Yoo, H-S Park, "A genetic algorithm for the vendormanaged inventory routing problem with lost sales, " Expert Syst Appl., vol. 53, pp. 149-159, 2016.

[43]U. Mehboob, J. Qadir, S. Ali, A. Vasilakos, " Genetic algorithms in wireless networking: techniques, applications, and issues. Soft Comput., vol. 20, pp. 2467-2501, 2016.

[44]A. Hiassat, A. Diabat, I. Rahwan, "A genetic algorithm approach for location-inventory-routing problem with perishable products," J Manuf Syst., vol. 42, pp. 93-103, 2017

[45]C.-C. Chen, C.-C. Tsao, Y.-C. Lin, C.-Y. Hsu, " Optimization of the sputtering process parameters of GZO films using the Grey-Taguchi method," Ceramics International, Vol. 36, no. 3, pp. 979-988, 2010. https://doi.org/10.1016/j.ceramint.2009.11.019. 